\title{
Automated Misalignment Compensating Interconnects Based on Self-Written Waveguides
}

\author{
Axel Günther, Sergej Schneider, Maher Rezem, Yixiao Wang, Uwe Gleissner, Thomas Hanemann, \\ Ludger Overmeyer, Eduard Reithmeier, Maik Rahlves, and Bernhard Roth
}

\begin{abstract}
Optical interconnects are the key components for integrated optics to link photonic integrated circuits or to connect external elements such as light sources and detectors. However, misalignment of the optical elements contained and its compensation is a remaining challenge for integrated optical devices. We present a novel method to establish rigid interconnects based on a 2-wavelength self-written waveguide process which automatically compensates for misalignment. We exemplarily demonstrate the capability of our process by writing interconnects between two multimode fibers as well as hot-embossed integrated polymer waveguides and a bare laser diode chip. The coupling efficiency of the interconnects obtained is analyzed with respect to misalignment. We found that coupling losses are as low as $1.3 \mathrm{~dB}$ if a lateral misalignment lies within a $10 \mu \mathrm{m}$ interval, which is achieved by commercially available pick-and-place machines. Our approach is easily combined with high-throughput techniques such as hot embossing and enables low-cost production of interconnects even for mass fabrication in future applications.
\end{abstract}

Index Terms-Optical coupling, optical interconnections, optical polymers, optical propagation, optical waveguides.

\section{INTRODUCTION}

D URING the last decade, optical information technology including signal generation, processing and transmission has gained increasing attention due to potentially higher transmission rates compared to its electronic counterpart. In addition, optical devices provide a high electromagnetic compatibility which is an important aspect in specific sensor applications in particular in a biomedical context [1]. For example, some

Manuscript received November 30, 2016; revised January 27, 2017 and April 5, 2017; accepted April 6, 2017. Date of publication April 6, 2017; date of current version May 15, 2017. This work was supported by the Deutsche Forschungsgemeinschaft within the Collaborative Research Center Transregio 123-Planar Optronic Systems. (Corresponding author: Axel Günther.)

A. Günther, S. Schneider, M. Rezem, M. Rahlves, and B. Roth are with the Hanover Centre for Optical Technologies, Leibniz Universität Hannover, Hanover 30167, Germany (e-mail: axel.guenther@hot.uni-hannover.de; schneider.s88@web.de; maher.rezem@hot.unihannover.de; maik.rahlves@hot. uni-hannover.de; bernhard.roth@hot.uni-hannover.de).

Y. Wang and L. Overmeyer are with the Institute of Transport and Automation Technology, Leibniz Universität Hannover, Hanover 30823, Germany (e-mail: yixiao.wangita.uni-hannover.de; ludger.overmeyerita.uni-hannover.de).

U. Gleissner and T. Hanemann are with the Institut für MikrosystemtechnikIMTEK, Albert-Ludwigs-Universitat Freiburg Institut fur Mikrosystemtechnik, Freiburg 79110, Germany (e-mail: uwe.gleissner@imtek.uni-freiburg.de; thomas.hanemann@imtek.de).

E. Reithmeier is with the Institute of Measurement and Automatic Control, Leibniz Universität Hannover, Hanover 30167, Germany (e-mail: eduard .reithmeier@imr.uni-hannover.de).

Color versions of one or more of the figures in this paper are available online at http://ieeexplore.iee.org.

Digital Object Identifier 10.1109/JLT.2017.2692305 sensors utilized for biomedical applications require light sources in the visible regime, such as presented in [2]. Optical data transfer as well as sensing networks are commonly based on optical waveguides. Classical materials for waveguide fabrication include silica $\left(\mathrm{SiO}_{2}\right)$, silicon nitride $\left(\mathrm{Si}_{3} \mathrm{~N}_{4}\right)$ but also polymer materials such as poly(methyl methacrylate) (PMMA). Especially, the latter material class is highly promising due to low material costs [3] and easy processability, which also enables stacking and bending of substrates [4]. However, for fabrication of complex integrated optical devices such as PIC's which include a large variety of passive and possibly active photonic devices [5], connecting different optical components is still a major challenge. Primary demands on interconnects naturally include low coupling losses but also cost effectiveness and the potential for a fast fabrication in an industrial context. Suitable coupling concepts not only depend on the specific devices to be connected but also on waveguide design. Prominent examples are interconnects between light sources such as laser diodes (LDs) or vertical-cavity surface emitting lasers (VCSELs) and light guiding structures such as embedded or surface mounted waveguides and waveguide arrays. Frequently used coupling structures for vertical coupling in the multi-mode regime are $45^{\circ}$-micro-mirrors as well as grating couplers for single or few mode applications, both being used to connect LDs and VCSELs. Coupling losses for polymer waveguides were reported in the range of $\approx 6-7 \mathrm{~dB}[6]$ and $\approx 2 \mathrm{~dB}$ [7] for grating and mirror couplers, respectively. In contrast, the coupling efficiencies reported for silicon photonics are significantly lower compared to their polymer based counterparts and typical coupling efficiencies were achieved down to $\leq 1 \mathrm{~dB}$ for grating couplers and $\approx 1 \mathrm{~dB}$ for mirrors [8]. The latter loss value of the polymer coupler relates to a rectangular waveguide core crosssection with a diameter of $85 \mu \mathrm{m} \times 100 \mu \mathrm{m}$. Connecting light sources such as LDs which launch light in lateral direction requires end-face coupling including suitable micro-optical components to enhance the coupling efficiency especially for smaller waveguides. A major source of error leading to increased coupling losses is misalignment between optical components due to positioning errors during fabrication such as in the case of pickand-place processes. Lateral positioning errors are typically in the order of a few microns and less than one degree for tilt and inclination. To connect two PICs and compensate for misalignment in silicon-on-insulator (SOI) applications, Lindenmann et al. created photonic wirebonds in SU-8 resist as chip interconnects [9]. Despite a high coupling efficiency and flexibility 
of the process, such approaches require accurate planning of the optical path and localization of features to be connected which is cumbersome.

In this work, we developed a process to create interconnects without the latter requirement and present, for the first time to our best knowledge, a fast and reliable process to connect horizontally emitting light sources and multi-mode waveguides by means of self written waveguides (SWW). It is capable of an automatic compensation for tilt, inclination and lateral shift errors and also forms a rigid mechanical link between both components, which is advantageous for future high-throughput applications. We combined SWW interconnects with hot embossed multi-mode waveguiding structures to demonstrate the potential of our approach to be integrated in a high throughput reel-to-reel process chain. The misalignment compensation done by Lindemann et al. was realized using single-mode waveguides. In this work the presented coupling elements are in the multi-mode regime. Concerning the results of Missinne et al., who demonstrated single-mode SWWs [10], we intend to downgrade this process for creating single-mode interconnects in a future work.

\section{Development of Self-Written WAVEguides}

The realization of self-written waveguides (SWW) by using photo polymerizing resins has been developed over the last years [11], [12] and was first presented by Frisken [13]. In [13], a UV-curing epoxy based monomer resin was utilized to create tapered guiding structures. Laser light was launched into a fiber with one of its end facets being placed inside the resin. The fiber tip acts as source point where the intensity distribution reaches its spatial maximum and the monomer starts to polymerize leading to a locally increased refractive index. Due to this increase, light is confined and guided within the polymerized area whereby a straight polymer waveguide forms and propagates into the uncured resin. A similar process has already been applied to write interconnects between two opposing fibers [14]. The process is schematically shown in Fig. 1(a). Depending on the monomer resin, the surrounding cladding material can also be cured by UV flood exposure after writing the SWW to achieve a rigid mechanical link between both fibers [14]. Recently, SWWs were also utilized for the fabrication of sensors [15], lasers [16] and coupling structures between VCSELs and photodiodes (PDs) [17]. However, a limiting factor for the fabrication of interconnects using SWW is the required wavelength of the light which usually lies in the UV region. This is especially restrictive when dealing with polymer waveguides or PICs due to their high absorption in the UV. In addition, using a single wavelength usually creates linear SWWs only and offsets and tilts between single components cannot be accounted for. A promising approach to overcome this drawback was recently presented by Yoshimura et al. [18]. Here, the UV-sensitive additives of the monomer resin were exchanged by an additive which is commonly used for photochemistry and enables polymerizing without an UV-source. In this work, as in [18], we utilized biacetyl(BA) as photoinitiator which is also known to support two-photon processes. In the context of SWWs, twophoton polymerization is referred to a process where a photon
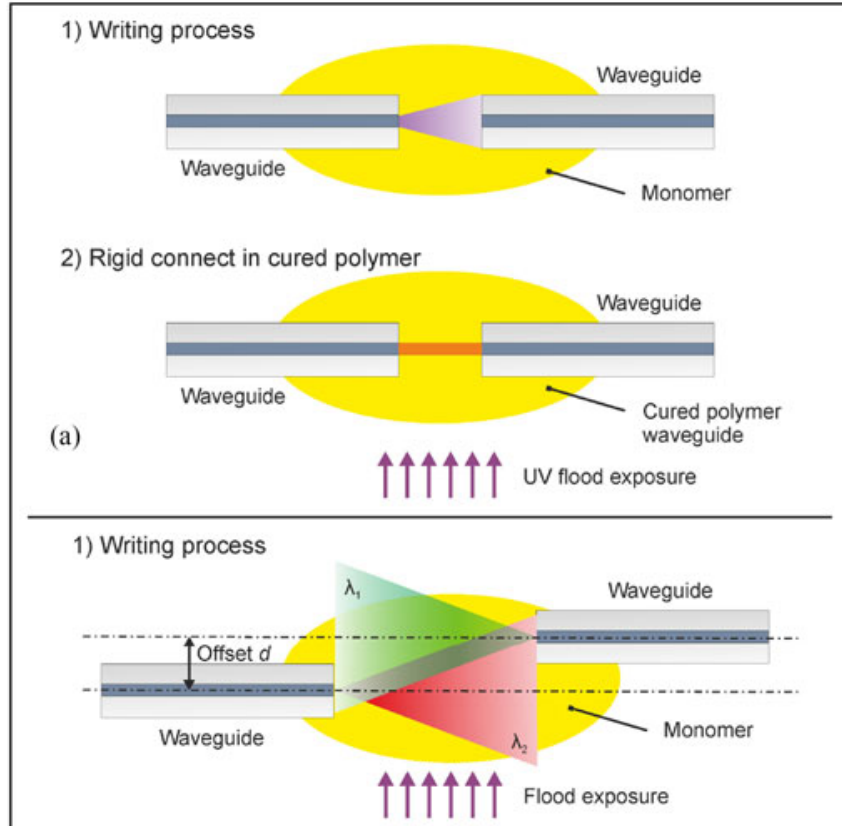

2) Rigid connect in cured polymer

(b)

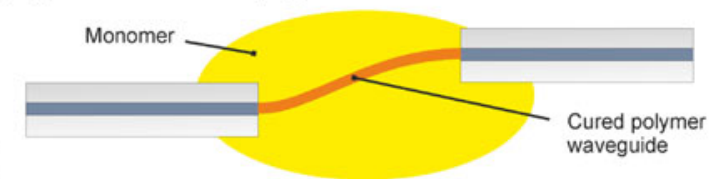

Fig. 1. Scheme of the writing process of (a) a SWW and (b) a 2-wavelengthSWW.

with wavelength $\lambda_{1}$ excites an electron from an energy level $S_{0}$ to $S_{n}$ (both spin singlet states) which transfers to a spin triplet $T_{1}$ state. A second photon with wavelength $\lambda_{2}$ further excites the electron from the energy level $T_{1}$ into a higher level $T_{m}$ which triggers the chemical reaction. In contrast to the work in [18], we introduced a third light source for flood exposure which can be switched on and off to trigger the polymerization processes. This effect might be due to a third energy level transfer which, however, needs to be investigated in detail in future work. This feature is a great advancement for practical applications compared to the previously demonstrated approaches and allows to selectively trigger a writing process between optical components such as laser diodes and waveguides which is demonstrated in this work.

\section{Preliminary Consideration}

\section{A. Preparation}

To connect two opposing fibers with an additional lateral offset, laser light at wavelengths of $448 \mathrm{~nm}$ and $780 \mathrm{~nm}$ was launched into each fiber, respectively. If the resin is applied on the gap between the fibers, a bent waveguide forms between the fiber cores due to two-wavelength absorption which is limited to the overlapping area of the two divergent beams launched into the resin from both fiber ends. Hence, the wavelength of the writing beams is not limited to the UV-spectrum. An 
additional advantage of this technique is, that the polymerization process starts only when a threshold energy is reached within the overlapping area. This allows for an external control of the process which can be accomplished by initiating and stopping the polymerization using an additional white light source, i.e. white light emitting LEDs. The process can also be used with only a single source beam and an additional external source. But, again, with this configuration only a straight SWW can be processed.

In this work, we applied a two-wavelength approach to create interconnects between LDs and embedded polymer waveguides without taking care of special alignment procedures. The general process for establishing a stable and low-loss optical interconnect between two fibers with misaligned optical axes is sketched in Fig. 1(b). First, the liquid resin is applied between both fiber ends, then, laser light at different wavelengths is launched through each fiber, respectively. Since polymerization requires light intensity above a certain threshold, an additional flood exposure was carried out using an external light source, as indicated in Fig. 1(b). An SWW based interconnect only forms in regions of the resin where the light cones of both fibers coincide and polymerization is triggered by the external light source. For connecting LDs with PICs, the LD itself serves as one of the light sources and can consequently be connected to a waveguide which carries the light of the second light source. A tremendous advantage of this process over established approaches is, that it leads to a self-guiding interconnect which automatically compensates for misalignment between optical components. Hence, this technique enables the production of PICs and other optical devices using standard pick-andplace machines with commonly observed placing tolerances of $\pm 10 \mu \mathrm{m}[19]$.

First, to fully evaluate the process sketched in Fig. 1(b) and also the created interconnects, we measured the attenuation of straight SWWs. Therefore, two OM2 gradient index fibers with core diameters of $50 \mu \mathrm{m}$ were positioned with opposing end facets and aligned optical axes. In the next step, the monomer mixture was applied onto the gap between both fibers. The monomer mixture consists of a matrix polymer, Syntholux (Synthopol, Buxtehude, Germany), which is a UV-curable epoxy acrylate and is composed of 80 weight- $\%$ tripropylene glycol dimethacrylat (TPGDA). A three weight-\% 2,3-butanedione (Sigma-Aldrich, Taufkirchen, Germany) served as UV-sensitive photo initiator. The reagents were compounded using a high speed mixer (Ultra-Turrax, T10, IKA, Germany) among standard conditions within a fume chamber until the mixture was completely homogeneous. Resulting air bubbles were removed by an ultrasonic bath. Then, the waveguide writing procedure was carried out, as described above.

\section{B. Attenuation Measurements}

To evaluate the attenuation $\alpha$ of a linear SWW, we realized SWWs with various length up to $2.5 \mathrm{~mm}$ using fiber coupled laser sources (Thorlabs, MCLS1-638 and MCLS1-850) at a wavelength of $638 \mathrm{~nm}$ and $850 \mathrm{~nm}$. During the writing process, laser light was launched from one fiber tip into the resin

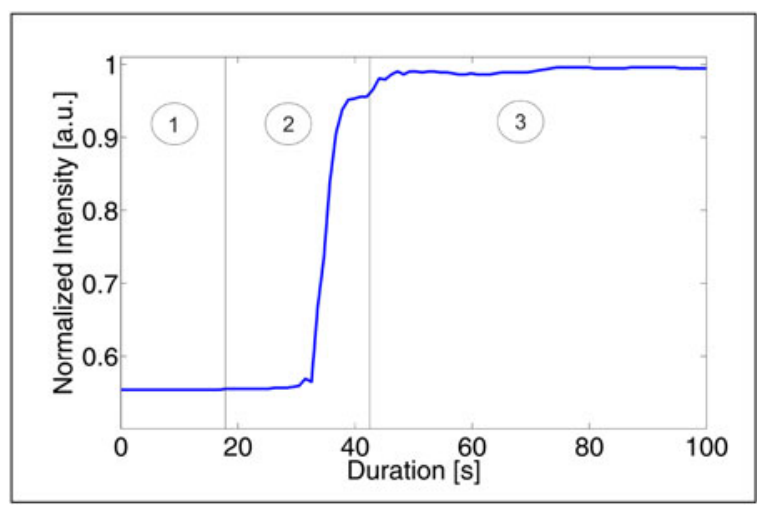

Fig. 2. Transmittance during the SWW writing process over a gap of $2.4 \mathrm{~mm}$.

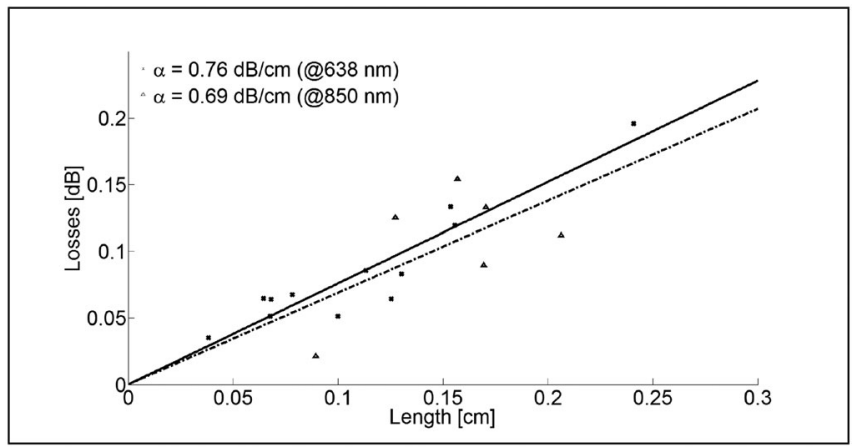

Fig. 3. Loss measurements on 2-wavelength-SWWs created in multiwavelength sensitive resin; measurements were carried out at a wavelength of $638 \mathrm{~nm}$ and $850 \mathrm{~nm}$

to ensure linear propagation of the SWW, while the other fiber was attached to a fiber coupled detector (Thorlabs, S120C) to monitor the transmitted intensity continuously. As external light source to control and trigger the polymerization process, a microscope LED ring illumination (SCHOTT, VisiLED ring light S80-55 BF) was employed. The SWWs created for attenuation measurements were fabricated using only a single laser source which was launched from a single fiber instead of two opposing fibers to simplify attenuation measurements. However, attenuation values obtained during these measurements yield the same results as would be expected for using two laser beams being launched into the monomer resin since the writing wavelength as well as writing power were chosen in the same range as used for all other writing experiments.

Fig. 2 shows the evolution of the transmitted intensity during the writing process using only a single source beam. At time (1), only the fiber coupled laser diode is emitting, during the time interval (2), the external LED was switched on to initiate the writing process and the SWW propagates into the resin until the connection between both fibers is completely established and the transmitted intensity reaches its maximum level (3). The duration of the writing process (2) can be controlled by the intensity of the external light source. In this case, the LED-ring illumination was at the maximum level, which corresponds to an illuminance of $\approx 200 \mathrm{klx}$ and increases the curing speed of the surrounding monomer. 


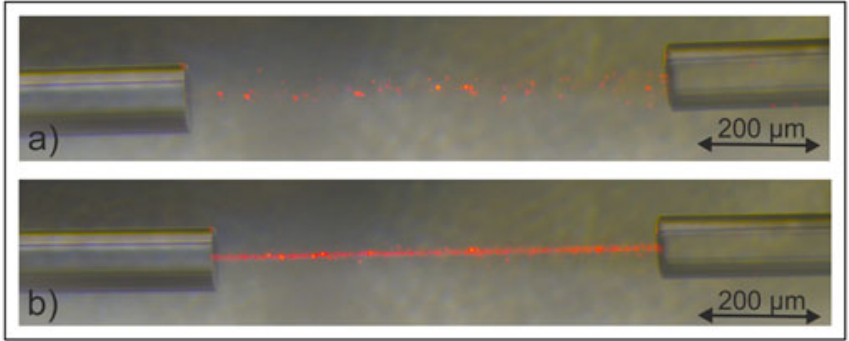

Fig. 4. Microscope image of two-wavelength SWW: (a) before initiation and (b) after completion of the writing process.

The attenuation values plotted in Fig. 3 are normalized to the intensity which was measured in direct butt-coupling fiber configuration. Thus, attenuation due to fiber based effects including Fresnel reflections is subtracted from the measurement results leading to an attenuation of $0 \mathrm{~dB}$ at a fiber length of $0 \mathrm{~cm}$ in Fig. 3. To calculate $\alpha$ from the measured intensities $I_{l}$ at a given length $l$, we determined the loss $L_{\mathrm{dB}}(l)=10 \cdot \log \left(I_{0} / I_{l}\right)$ for each length $l$. $I_{0}$ corresponds to the initial intensity value when both fibers are as close to each other as possible and the monomer has already been applied which corresponds to a length of $l=0$. The attenuation is obtained by means of a linear fit, as shown in Fig. 3. The gradients of the fit in Fig. 3 yield attenuations of $\alpha=(0.76 \pm 0.16) \mathrm{dB} / \mathrm{cm}$ at $638 \mathrm{~nm}$ and $\alpha=(0.69 \pm 0.31) \mathrm{dB} / \mathrm{cm}$ at $850 \mathrm{~nm}$. The large variation of the intensity values of the measurement at $850 \mathrm{~nm}$ are due to the measuring procedure. Because the additives used in the monomer mixtures are not suitable for $850 \mathrm{~nm}$, the writing process was done with the $638 \mathrm{~nm}$ source. After the writing process, the fiber was reconnected to the $850 \mathrm{~nm}$ port. Naturally, it would be desirable to utilize a writing wavelength of $850 \mathrm{~nm}$ or even larger to connect sources which are operated at classical telecommunication wavelength. The objective of this work was a proof-of-principle which enables the connection of laser diodes emitting in the visible spectral range (VIS). To expand this technique towards the near infrared (NIR) regime, different photoinitiators which start the polymerization process at higher wavelengths are required which will be in the focus in future work [20].

A microscope image of an established SWW connection between two fibers is shown in Fig. 4. Before the creation of a SWW, light distributes in a divergent cone inside the resin, as shown in Fig. 4(a). After the connection between the two fibers was realized, see Fig. 4(b), the distributed light beam is confined within the interconnect and is observable as slim red line.

\section{Misalignment Compensation}

To demonstrate that our process allows for compensation of misalignment between optical components by automatically tracing its own path through the resin, we fabricated interconnects between two misaligned opposing OM2 fibers. The end facets of the fibers were $1 \mathrm{~mm}$ apart along their optical axis and placed on a linear translation stage (Elliot Scientific, MDE122) to adjust a lateral offset $d$, as shown in Fig. 1(b). The writing process was realized by using two emitting fibers launching light at wavelengths of $638 \mathrm{~nm}$ and $660 \mathrm{~nm}$ into the resin,

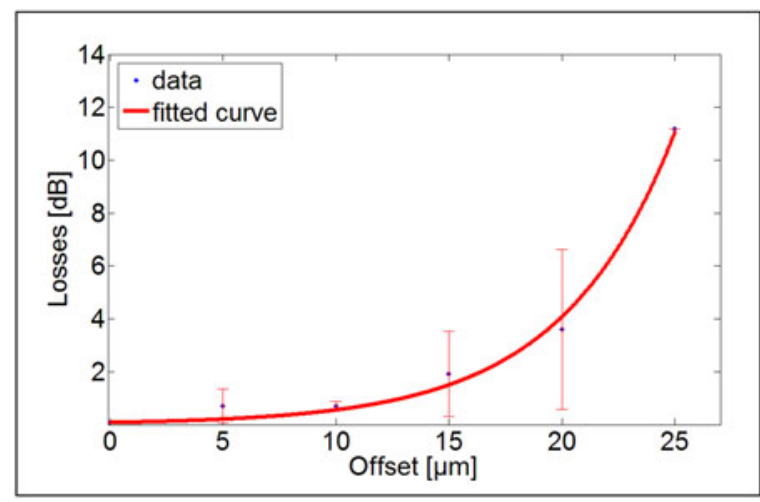

Fig. 5. Loss measurements for different offsets $d$ between the optical axis of two fibers.

respectively. Again, the external light source was utilized to trigger the polymerization process. Sufficiently accurate positioning was ensured using manually adjustable high precision stages with a positioning tolerance of $\pm 20 \mu \mathrm{m}$ over a total travel distance of $2 \mathrm{~mm}$.

We fabricated SWWs for different offsets $d=0 \ldots 25 \mu \mathrm{m}$ in $5 \mu \mathrm{m}$ increments and determined the loss of the interconnect as described previously. The loss measurement showed total coupling losses less than $1.3 \mathrm{~dB}$ and $3 \mathrm{~dB}$ for offsets $d \leq 10 \mu \mathrm{m}$ and $d \leq 20 \mu \mathrm{m}$, respectively, as shown in Fig. 5. At an offset $d=25 \mu \mathrm{m}$ a significant increase in coupling loss to $11 \mathrm{~dB}$ was observed, which can be attributed to the limited cone angle of light emerging from both cores of the fibers. To demonstrate that the SWW interconnect drastically enhances the coupling efficiency compared to the same fiber configuration without SWW interconnect, we measured the resulting coupling losses for both cases. The initial intensity value after butt coupling of the two fibers inside the applied monomer droplet was $I_{0}=6 \mathrm{~mW}$. After adjusting the distance to $1 \mathrm{~mm}$ and a gap size of $10 \mu \mathrm{m}$ the measured power decreased to $I_{\text {dist }}=1.7 \mathrm{~mW}$. Consequently the SWW was created whereby the transmitted power increased up to $4.7 \mathrm{~mW}$ which corresponds to an attenuation of $\approx 1 \mathrm{~dB}$. Hence, the interconnect drastically increased the transmitted intensity. If the offset $d$ exceeds a certain value, both light cones do not sufficiently overlap inside the resin, leading to an undeveloped interconnect as is observable at $d=25 \mu \mathrm{m}$. Additional measurements based on the refractive near field method yield a refractive index difference between core and cured cladding of $\Delta n=4 \cdot 10^{-4}$. This small difference leads to increasing losses for higher offsets. However, when connecting optical components which were positioned using pick-and-place automates placing tolerances are less than $10 \mu \mathrm{m}$ and, thus, the maximal attenuation is $1.3 \mathrm{~dB}$. This value corresponds to a coupling efficiency of $\approx 74 \%$, which has been unrivaled yet for connecting two optical components with such a misalignment and structure size, to the best of our knowledge.

\section{RESULTS}

To demonstrate the capability of our process to connect different optical components, we exemplary fabricated an interconnect between an LD (LD chip-650-P5, Roithner Lasertechnik) 


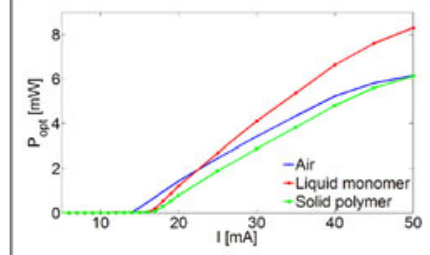

(a)

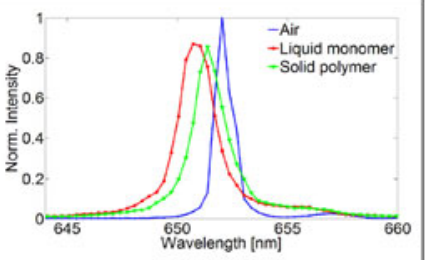

(b)
Fig. 6. Changes of the (a) P-I-characteristic and (b) the emitted spectrum of the laser diode after applying and curing of the monomer droplet.

and a hot embossed embedded waveguide. The bare LD chip was bonded onto a FR4 circuit board before writing the interconnect [21]. The emitted wavelength of the LD chip ranges between $650 \mathrm{~nm}$ and $660 \mathrm{~nm}$ depending on its temperature.

For creating the SWW coupling structure, the monomer droplet has to applied on the LD. To evaluate the influence of the monomer on characteristics of the LD, the lasing threshold and spectrum were measured using the same equipment as described previously. The results are shown in Fig. 6.

It shows the measured optical power and spectrum of the laser diode in air, surrounded by the liquid resin and after the curing process. In Fig. 6(a), a shift of the lasing threshold of the LD is observable. This effect is due to a change in the surrounding refractive index caused by applying the monomer which decreases the reflectivity of the end facets of the LD-resonator. Thus, a higher pump current is required to establish stimulated emission inside the LD. The optical power and spectrum were measured using a fiber-coupled spectrometer (Avantes, Avaspec-3648) and detector (Thorlabs, S120-C) attached to a $200 \mu \mathrm{m}$ fiber which was placed in front of the diode. Curing of the monomer leads to a misalignment of the fiber which reduces the measured optical power for the cured state of the monomer in Fig. 6(a). The recorded spectra in Fig. 6(b) which were measured at an LD driving current of $20 \mathrm{~mA}$ show a peak broadening and shift of the emitted wavelength. The increase of the full width at half maximum (FWHM) from $F W H M_{\text {air }}=0.95 \mathrm{~nm}$ in air to $F W H M_{\text {monomer }}=1.35 \mathrm{~nm}$ and $F W H M_{\text {polymer }}=1.7 \mathrm{~nm}$, is caused by a decrease of the resonator's Q-factor due to application of the monomer. The shift of the center wavelength towards a shorter one from $\lambda_{\text {air }}=652 \mathrm{~nm}$ to $\lambda_{\text {monomer }}=650.7 \mathrm{~nm}$ and $\lambda_{\text {polymer }}=651.4 \mathrm{~nm}$, respectively, is due to a better heat transfer as result of an increased heat conduction in the liquid monomer resin. In addition, the influence of the monomer on the slope-efficiency yields $13.6 \%$ for the LD in air, $19.0 \%$ after applying the monomer and $14.2 \%$ after the resin was cured. The change in slope-efficiency is also caused by the monomer which changes the surrounding refractive index and thus the reflectivity of the resonator. To create coupling structures suitable for polymer based PICs, a sample containing polymer waveguides was placed in front of the LD manually. The waveguides were fabricated in a poly(methyl methacrylate) (99524 GT Plexiglas, Evonik) substrate by hot embossing. After embossing of trenches in PMMA which serves as cladding, NOA68 (Norland) optical adhesive acting as the core material was distributed on the substrate through a doctor blading process and UV cured

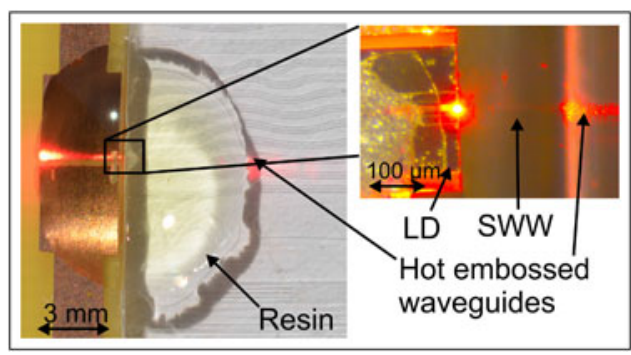

Fig. 7. Image of the SWW interconnect between LD and hot embossed waveguides.

subsequently. For a detailed description of the fabrication process we refer to [22]. Before initiating the writing process, a coarse adjustment of the waveguide in front of the LD was carried out with aid of standard linear positioning stages (Elliot Scientific) to mimic positioning by pick-and-place machines. The positioning was assisted by monitoring the light intensity being transmitted through the waveguides using a beam profiler (Ophir, SP620U). After this, a monomer resin droplet was applied between the LD and the waveguides. To create a selfguiding SWW interconnect, the LD was connected to a power supply (ILX Lightwave, LDX3412). The second laser source, which substitutes the beam profiler and runs at a wavelength of $638 \mathrm{~nm}$, was launched into the polymer waveguide to be connected by fiber butt coupling. Writing was initiated by exposure using the external light source as during the previous experiments.

Fig. 7 shows an image of the fabricated optical device containing the LD, an SWW and polymer waveguides. To determine the coupling losses of the interconnect, we measured the output power of the LD at the side of the chip facing the waveguide yielding $P_{0}=3.5 \mathrm{~mW}$. A power of $P=0.7 \mathrm{~mW}$ was measured after propagating through a waveguide with a length of $1 \mathrm{~cm}$ giving an optical attenuation of $\alpha=0.74 \mathrm{~dB} / \mathrm{cm}$. Consequently, we achieved a coupling efficiency of $\left(P \cdot 10^{\frac{\alpha}{10}}\right) / P_{0} \cdot 100=$ $23.7 \%$ which relates to a coupling loss of $6.2 \mathrm{~dB}$.

\section{CONCLUSION}

In summary, we presented a two-wavelength self-written waveguide (SWW) process which is suitable for realization of optical interconnects. The process relies on a custom-made monomer mixture which is applied on a gap between the components to be connected. If two divergent light beams are launched from both optical components, an overlapping region of both cones forms inside the monomer with increased intensity. An external light source is then used to trigger the formation of the interconnects which traces its own way along the region of maximal intensity. This enables the connection of a light source and a waveguide with a displacement between their optical axes. Attenuation measurements on linear SWW yielded values of $(0.76 \pm 0.16) \mathrm{dB} / \mathrm{cm}$. To analyze the capability of the presented process to compensate for misalignment, interconnects between two multi-mode waveguides with misaligned optical axes were created. We found that optical losses are as low as $1.3 \mathrm{~dB}$ and $3 \mathrm{~dB}$ for offsets between both axes of 
$10 \mu \mathrm{m}$ and $20 \mu \mathrm{m}$, respectively. Hence, the achieved losses are sufficiently low considering commonly observed misalignment errors in pick-and-place processes which are less than $10 \mu \mathrm{m}$. The presented process was demonstrated in combination with hot embossing to demonstrate that it lends itself for the large scale and high-throughput fabrication of interconnects including an automatic compensation of misalignment. The process is especially suited for commercial processes and will enhance the fabrication of polymer optical circuit boards in applications ranging from optical computing to optical sensing.

\section{REFERENCES}

[1] N. K. Guimard, N. Gomez, and C. E. Schmidt, "Conducting polymers in biomedical engineering," Prog. Polymer Sci., vol. 32 nos. 8/9, pp. 876921, May 2007.

[2] J. Lou, L. Tong, and Z. Ye, "Modeling of silica nanowires for optical sensing," Opt. Express vol. 13, no. 6, pp. 2135-2140, Mar. 2005

[3] T. Yamashita, M. Kagami, and H. Ito, "Waveguide shape control and loss properties of light-induced self-written (LISW) optical waveguides," $J$. Lightw. Technol., vol. 20, no. 8, pp. 1556-1562, Apr. 2002.

[4] S. Lehmacher and A. Neyer, "Integration of polymer optical waveguides into printed circuit boards," Electron. Lett., vol. 36, no. 12, pp. 1052-1053, Jun. 2000

[5] C. Zhang, S. Zhang, J. D. Peters, and J. E. Bowers, " $8 \times 8 \times 40$ GBPS fully integrated silicon photonic network on chip," Optica, vol. 3, no. 7, pp. 785-786, Jul. 2016.

[6] R. Bruck et al., "Flexible thin-film polymer waveguides fabricated in an industrial roll-to-roll process," Appl. Opt., vol. 52, no. 19, pp. 4510-4514, Jul. 2013.

[7] M. Immonen, M. Karppinen, and J. K. Kivilahti, "Fabrication and characterization of polymer optical waveguides with integrated micromirrors for three-dimensional board-level optical interconnects," IEEE Trans. Electron. Packag. Manuf., vol. 28, no. 4, pp. 304-311, Oct. 2005

[8] S. Lardenois, D. Pascal, L. Vivien, E. Cassan, and S. Laval, "Low-loss submicrometer silicon-on-insulator RIB waveguides and corner mirrors," Opt. Lett., vol. 28, no. 13, pp. 1150-1152, Jul. 2003.

[9] N. Lindenmann et al., "Photonic wire bonding: A novel concept for chipscale interconnects," Opt. Express, vol. 20, no. 16, pp. 17667-17677, Jul. 2012.

[10] J. Missine et al., "Self-written waveguides for field-installable fiber connectors," in Proc. Adv. Photon. Conf., Jul. 2013, Paper JT3A.7.

[11] S. Shoji, S. Kawata, A. A. Sukhorukov, and Y. S. Kivshar, "Self-written waveguides in photopolymerizable resins," Opt. Lett., vol. 27, no. 3, pp. 185-187, Feb. 2002.

[12] M. Kagami, T. Yamashita, and H. Ito, "Light-induced self-written threedimensional optical waveguide," Appl. Phys. Lett., vol. 79, no. 8, pp. 10791081, Aug. 2001.

[13] S. J. Frisken, "Light-induced optical waveguide uptapers," Opt. Lett., vol. 18, no. 13, pp. 1035-1037, Jul. 1993.

[14] A. Günther et al., "Cladded self-written multimode step-index waveguides using a one-polymer approach," Opt. Lett., vol. 40, no. 8, pp. 1830-1833, Apr. 2015.

[15] Y. J. Song and K. J. Peters, "A self-repairing polymer waveguide sensor," Smart Mater. Struct., vol. 20, no. 6, May 2011, Art. no. 065005.

[16] K. Yamashita, A. Kitanobou, M. Ito, E. Fukuzawa, and K. Oe, "Solid-state organic laser using self-written active waveguide with in-line Fabry-Perot cavity," Appl. Phys. Lett., vol. 92, no. 14, Apr. 2008, Art. no. 143305.

[17] M. J. Kwack, M. Kanda, O. Mikami, M. Yonemura, and M. Kagami, " $180^{\circ}$ light path conversion device with tapered self-written waveguide for optical interconnection," IEEE Photon. Technol. Lett., vol. 22, no. 15, pp. 1126-1128, Aug. 2010.

[18] T. Yoshimura, D. Takeda, T. Sato, Y. Kinugasa, and H. Nawata, "Polymer waveguides self-organized by two-photon photochemistry for self-aligned optical couplings with wide misalignment tolerances," Opt. Commun., vol. 362, pp. 81-86, Jun. 2016.

[19] J. W. Nah, Y. Martin, S. Kamlapurkar, S. Engelmann, R. L. Bruce, and T. Barwicz, "Flip chip assembly with sub-micron 3D re-alignment via solder surface tension," in Proc. IEEE Electron. Compon. Technol. Conf., May 2015, pp. 35-40.
[20] N. Corrigan, J. Xu, and C. Boyer, "A photoinitiation system for conventional and controlled radical polymerization at visible and NIR wavelengths," ACS Macromolecules, vol. 49, no. 9, pp. 3274-3285, Apr. 2016.

[21] Y. Wang and L. Overmeyer, "Chip-level packaging of edge-emitting laser diodes onto low-cost transparent polymer substrates using optodic bonding," IEEE Trans. Compon., Packag., Manuf. Technol., vol. 6, no. 5 , pp. 667-674, Apr. 2016.

[22] M. Rezem, A. Günther, M. Rahlves, B. Roth, and E. Reithmeier, "Low-cost fabrication of all-polymer components for integrated photonics," J. Lightw. Technol., vol. 35, no. 2, pp. 299-308, Jan. 2017, doi 10.1109/JLT.2016.2639740.

Axel Günther received the B.Eng. degree from the University of Applied Sciences of Zwickau, Zwickau, Germany, in 2011, and the M.Sc. degree in optical technologies from the Leibniz Universität Hannover, Hanover, Germany, in 2013. He is currently working toward the Ph.D. degree at the Hanover Centre for Optical Technologies, Leibniz Universität Hanover. His research interest include polymer-based micro optics, optical technology for life sciences, and biophotonics.

Sergej Schneider received the B.Sc. degree in nanostructure and molecular sciences from the University of Kassel, Kassel, Germany, in 2014. He is currently working toward the Master's degree in optical technologies at the Leibniz Universität Hannover, Hanover, Germany. Since 2016, he has been working as a Student Research Assistant at the Hanover Centre for Optical Technologies, Leibniz Universität Hanover.

Maher Rezem received the Diploma degree in electrical engineering from the Karlsruhe Institute of Technology, Karlsruhe, Germany, in 2012. He is currently working toward the Ph.D. degree in engineering at the Hanover Center for Optical Technologies, Leibniz University of Hanover, Hanover, Germany. He is currently working in the field of polymer optics manufacturing by means of hot embossing and UV-imprinting.

Yixiao Wang received the Master's degree in electrical engineering with a focus on automation technology from the Leibniz Universität Hannover, Hanover, Germany, in 2011. Since 2012, she has been a Research Associate in the Institute for Transport and Automation Technology, Leibniz Universität Hanover. She concentrates on the development of the packaging processes of optoelectronics on low-cost polymer foils. Her research interests include the low-temperature flip chip bonding technology, optoelectronic packaging on flexible polymer foils as well as optical alignment and coupling of optoelectronic components in planar optronic systems.

Uwe Gleissner received the B.Sc. and M.Sc. degrees in microsystems engineering from the Department of Microsystems Engineering, University of Freiburg, Freiburg im Breisgau, Germany, in 2009 and 2012, respectively. He is currently working at the University of Freiburg within the Collaborative Research Center Transregio 123-Planar Optronic Systems, which is supported by the German Research Foundation. His current research focuses on the development of optically and rheologically tailored polymers for integrated optics.

Thomas Hanemann received the Doctorate degree in physical chemistry from Technische Universitt Darmstadt, Darmstadt, Germany, in 1993. He was a Visiting Scientist at the IBM Almaden Research Center San José in 1994-1995. $\mathrm{He}$ was the Head of the Laboratory for Materials Processing, Department of Microsystems Engineering, University of Freiburg, in 2011. His research interests include nonsilicon materials in microsystems technologies, polymernanomaterial composites, replication technology, and optical materials. 
Ludger Overmeyer received the Diplom-Ingenieur degree in electrical engineering from the Leibniz Universität Hannover, Hanover, Germany, in 1991. From 1991 to 1997, he worked as a Research Associate and the Department Leader of the Laser Zentrum. He also worked as a Leader for research and development at the Mühlbauer AG, Roding, Germany. Since 2002, he has been a Professor and the Chair of the Institute of Transport and Automation Technology, Leibniz University of Hanover, Hanover, Germany. He has been the Executive Director of the Institute of Integrated Production $\mathrm{GmbH}$ (IPH) since 2007 and a member of the executive board of the Laser Zentrum Hanover since 2010. He has authored and coauthored more than 100 conference papers, journal papers, and book chapters. His research interests include transport technology, automation technology, laser materials processing, optoelectronic packaging, and planar optronic sensor systems.

Eduard Reithmeier received the Diploma degree in mechanical engineer ing, the Diploma degree in mathematics, and the Ph.D. degree in mechanical engineering, all from the Technical University of Munich, Munich, Germany, in 1977, 1979, and 1989, respectively. From 1992 to 1996, he was the Technical Director of the Business Divisions Automation and Medical Engineering with Bodenseewerk Geraetetechnik GmbH, Ueberlingen, Germany. Since 1996, he has been a Professor of Mechanical Engineering and the Director of the Institute for Measurement and Control with Leibniz University of Hanover, Hanover, Germany. His research interests include industrial and medical imaging, acoustics, production measurement technology, and control theory.
Maik Rahlves received the Graduate degree in physics from the Carl-vonOssietzky University of Oldenburg, Oldenburg, Germany, in 2006, and the $\mathrm{Ph}$.D. degree in mechanical engineering from the Leibniz University of Hanover, Hanover, Germany, in 2011. From 2006 to 2009, he was a Research Associate at the Institute of Measurement and Automatic Control, Leibniz University of Hanover, where he focused on confocal microscopy and interferometry in surface metrology. Since 2009, he has been with the Hanover Centre for Optical Technologies, Leibniz University of Hanover. Since 2011, he has been the Head of the Applied Optics Group, Hanover Centre for Optical Technologies. His research interests include optical metrology, holography, and polymer-based microoptics.

Bernhard Roth received the Ph.D. degree in atomic and particle physics from University of Bielefeld, Bielefeld, Germany, in 2001. From 2002 to 2007, he was a Research Group Leader at the University of Duesseldorf, where he received the State Doctorate (Habilitation) in experimental quantum optics in 2007. Since 2012, he has been a Scientific and Managing Director of the Hanover Centre for Optical Technologies, Hannover, Germany, and since 2014, a Professor of physics at the Leibniz University Hanover, Hannover, Germany. His scientific activities include applied and fundamental research in laser development and spectroscopy, polymer optical sensing as well as optical technology for illumination, information technology, and the life sciences. 